\title{
Co-Designing Smartphone Notifications According to the Clinical Routine of Cancer Patients
}

\author{
Panos BONOTIS ${ }^{\mathrm{a}, 1}$, Fatima SCHERA ${ }^{\mathrm{b}}$, Christina KARAMANIDOU ${ }^{\mathrm{a}}$, Lydia \\ SCARFO $^{\mathrm{c}}$, Charalambos PONTIKOGLOU ${ }^{\mathrm{d}}$ and Christos MARAMIS ${ }^{\mathrm{a}}$ \\ anstitute of Applied Biosciences, Centre for Research \& Development Hellas, Thermi \\ Thessaloniki, Greece \\ ${ }^{\mathrm{b}}$ Institute for Biomedical Engineering, Fraunhofer, Germany \\ ${ }^{\mathrm{c}}$ Universita Vita-Salute San Raffaele and IRCCS Ospedale San Raffaele, Milan, Italy \\ ${ }^{\mathrm{d}}$ Department of Hematology and Oncology, University Hospital of Heraklion, Greece
}

\begin{abstract}
Although smartphone-based notifications offer a promising tool to support patient engagement and data collection via mobile health apps, attention must be given to the burden caused by frequent notifications and notification timing. This study presents a personalized mobile notification scheme, designed and developed to optimize reachability, and thus data collection from patients. Engineers, psychologists, oncologists, and patients were involved in various stages of a codesign approach and the presented implementation is currently used in the context of a clinical study.
\end{abstract}

Keywords. mHealth, palliative care, notification fatigue, eHealth, cancer

\section{Introduction}

Smartphone notifications are typically used to remind tasks and raise data collection prompts to end-users/patients. Considering the fact that users can receive in excess of 50 notifications per day from a variety of apps, and that different message formats (i.e. email, SMS, "push" notifications etc.) may have adverse effects on desired behavior compared to the use of just one message type, it is evident that notification delivery should be carefully designed [1] to reduce burden, a.k.a. notification fatigue. This becomes more clear considering the extensive research indicating that apps may be quickly discarded if notifications are perceived to be irritating or intrusive [2].

In this paper, we present a mobile notification scheme that was conceptualized, designed, and deployed as part of a mobile app used in the context of the MyPal project's ${ }^{2}$ adult study [3] and is used to prompt patient input along the paradigm of electronic Patient Reported Outcomes (ePROs) for palliative care [4] and the delivery of personalized motivational messages.

\footnotetext{
${ }^{1}$ Corresponding Author, Panos Bonotis, eHealth Lab, INAB-CERTH, 6th Km. Charilaou-Thermi Road, Thermi, Thessaloniki, Greece; E-mail: pbonotis@certh.gr.

2 The MyPal project has received funding from the European Union's Horizon 2020 research and innovation programme under grant agreement No 825872, https://mypal-project.eu/.
} 


\section{Methods \& Results}

In order to align ePRO notifications timing with the clinical routine of patients and reduce "notification fatigue", a participatory design approach was applied, organized in 3 distinct design phases: (a) a panel discussion and focus group session with stakeholders (healthcare professionals, patients) during which the notification scheme requirements were identified, (b) the initial design phase ( 2 engineers and a psychologist), where it was decided to merge each day's notifications (ePRO prompts, motivational messages) as one notification and the frequency of the notifications was defined as 4-5 per week, and (c) the definition of the in-day timeslots for the notifications to be presented. Considering the specific disposition of cancer patients during or after treatments, in order to define the in-day slots, oncologists were actively involved to identify patient communication patterns: interviews were performed, with chronic lymphocytic leukemia (CLL) and a myelodysplastic syndrome (MDS) oncologists to initially document the clinical routine of patients and these findings were confirmed via a survey by 6 independent physicians. The survey responses served in the design of a set of priority rules for presenting a notification in a day. To this end, a day was represented by a 24size matrix (24 hours in a day) depicting the users' "quiet hours" (default 23:00-7:00) and "preferred notification time" (user-entered), also aligned with the patient's appointment and treatment schedule. The app calculates the optimum slot based on a decision tree defined by oncologists which was later also validated by patient advocates.

\section{Conclusions}

Currently, the presented notification scheme is operating as part of the MyPal adult study and is currently being evaluated in the context of the MyPal clinical trials.

\section{Acknowledgments}

The authors would like to thank Dr. Pantelis Natsiavas, the technical coordinator of the MyPal project for his contribution in the presented poster manuscript.

\section{References}

[1] M. Czerwinski, et. al, Challenges for designing notifications for affective computing systems, UbiComp 2016 Adjun. - Proc. 2016 ACM Int. Jt. Conf. Pervasive Ubiquitous Comput. (2016) 1554-1559. doi:10.1145/2968219.2968548.

[2] L.G. Morrison, et. al, The Effect of Timing and Frequency of Push Notifications on Usage of a Smartphone-Based Stress Management Intervention: An Exploratory Trial, PLoS One. 12 (2017). doi:10.1371/JOURNAL.PONE.0169162.

[3] C. Karamanidou, et. al, Electronic Patient-Reported Outcome-Based Interventions for Palliative Cancer Care: A Systematic and Mapping Review., JCO Clin. Cancer Informatics. 4 (2020) 647-656. doi:10.1200/CCI.20.00015.

[4] C. Maramis, et. al, Using Electronic Patient Reported Outcomes to Foster Palliative Cancer Care: The MyPal Approach, in: Proc. - 2019 IEEE 19th Int. Conf. Bioinforma. Bioeng. BIBE 2019, Institute of Electrical and Electronics Engineers Inc., 2019: pp. 405-409. doi:10.1109/BIBE.2019.00079. 